Clío Américal Vol. 12, No. 24 - 2018 / 137 - 148

DOI: http://dx:10.21676/23897848.2818

\title{
EL FENÓMENO DE LOS FOOD TRUCKS EN LA CIUDAD DE BARRANQUILLA, ¿ÉXITO, FRACASO O UNA SIMPLE MODA?
}

\section{THE FOOD TRUCKS PHENOMENON IN THE CITY OF BARRANQUILLA, SUCCESS, FAILURE OR A SIMPLE TREND?}

\section{Alex José Lama-Acosta ${ }^{1}$, Martha Cecilia Ucros-Berrio ${ }^{2}$ y $^{(1)}$ Harold Silva-Guerra $^{3}(\mathbb{D}$}

Tipología: Artículo de investigación científica y tecnológica

Para citar este artículo: Lama, A. A., Ucros, B. M. y Silva, G. H. (2018). El fenómeno de los food trucks en la ciudad de Barranquilla, ¿éxito, fracaso o una simple moda?. Clío América, 12(24), 137 - 148. doi: http://dx.doi.org/10.21676/23897848.2818

Recibido en agosto 14 de 2018

Aceptado en 01 de noviembre de 2018

Publicado en línea en 28 de noviembre de 2018

\section{RESUMEN}

Este artículo presenta resultados del proyecto de investigación "El fenómeno de los food trucks en la ciudad de barranquilla, ¿éxito, fracaso o una simple moda?, que persigue analizar este suceso en la ciudad y sus alcances como negocio. Se desarrolló una investigación de tipo mixta, basada en las experiencias propias de comensales que han tenido la oportunidad de visitar los food trucks de la ciudad. El desarrollo metodológico de la investigación se centró en diferentes herramientas de apoyo, tales como: Grupos focales, cuestionarios y encuestas de forma abierta, entrevistas no estructuradas y observaciones no estructuradas. En Barranquilla, los food trucks fueron una moda, y que al no cumplir con la esencia de ser móvil e itinerante, fueron un boom que lamentablemente duró muy poco. Como conclusión consideran los clientes, que esta experiencia fracasó por falta de innovación y que los food truck se convirtieron en un negocio de copiar y repetir donde no había propuestas diferenciadoras. Se difundió rápidamente y finalmente se encuentra en decadencia o casi que en extinción.

Palabras clave: food truck - comida callejera - clientes - gastronomía - Barranquilla.

JEL: M10 M13, M31, O31.

\section{ABSTRACT}

This article presents results of the research project "The phenomenon of food trucks in the city of Barranquilla, success, failure or a simple trend?" that seeks to analyze this event in the city and its scope as a business. A mixed research was developed based on the experiences of diners who have had the opportunity to visit the food trucks of the city. The methodological development of the research focused on different support tools such as: focus groups, open questionnaires and surveys, unstructured interviews and unstructured observations. In Barranquilla, food trucks were a fad, and when they stopped being mobile and itinerant, they became a boom that, unfortunately, lasted very little. In conclusion, clients believe this experience failed due to a lack of innovation, and the fact that all food trucks copied and repeated the same business model; there were no differentiating proposals. The trend spread quickly, but now it is in decline, or about to become extinct.

Keywords: food truck - street food - clients - gastronomy - Barranquilla.

1 Universidad del Norte. Colombia. Email: alama@uninorte.edu.co ORCID: https://orcid.org/0000-0002-5460-6935

2 Universidad del Norte. Colombia. Email: marucros@gmail.com ORCID: https://orcid.org/0000-0001-6219-2536

3 Universidad del Norte Escuela de Negocios. Colombia. Email: hsilva@uninorte.edu.co ORCID: https://orcid.org/0000-0002-6203-3911 


\section{INTRODUCCIÓN}

La comida rápida surge de la necesidad de las personas en las grandes urbes, por ejemplo en Japón o Nueva York, con la venta de sushi o los carros de ruedas empujados en las calles que data desde 1691. La usanza de la comida callejera y los comedores móviles han hecho parte de la cultura del consumo de los americanos desde finales del siglo XVII.

Barcelona Foodie Guide (2015) asevera que Charles Goodnight, revolucionó la ganadería en Estados Unidos, concretamente en Texas cuando compró un viejo vagón para llevar alimentos, agua y otros insumos ante el casi total aislamiento que sufrían los ganaderos de esa región.

Díaz (2015) afirma que en 1936 Raúl Martínez acondicionó un viejo camión que era utilizado para la venta de helados en algo que se conoce como lo más parecido a un restaurante móvil de la actualidad, convirtiéndose en un verdadero pionero de esta actividad.

Newman (2018) destaca que el ejemplo de Martínez fue creciendo, a través de innovaciones y a partir del 2009 hizo su aparición, poco a poco, en el Canadá y en Miami, donde contagió miles de empleados de factorías, que optaban por este modelo no solo por la agilidad del sistema, sino porque no necesitaba pagar propinas, lo cual rebajaba sus costos.

La comida callejera, o street food, siempre ha existido en la ciudad, en los pequeños puestos de arepas, de perros calientes y tradicionales chuzos que se popularizan sobre todo para los días de carnavales en la ciudad. Este tipo de negocio ambulante, de esquina, que aparecen cada tarde en la ciudad y desaparecen entrada la noche, siguen allí y al parecer no dejarán de estar, pues son un fenómeno aparte y diferente del nuevo street food, un poco más refinado, que apareció recientemente en la ciudad con los food trucks, moda estadounidense que quiso llegar a tomarse las calles de Barranquilla.

Según Ramírez (2018):

En los últimos años, la comida que se cataloga como callejera ha trascendido a conceptos más elaborados y recetas que superan lo simple y básico. Hablar de comida callejera o street food es hacer referencia a una tendencia que viene tomando fuerza en el mundo desde hace algunos años (p. 1).

Desde el año 2010, este creciente fenómeno toma fuerza día a día en Colombia y en Barranquilla, donde no pasó desapercibido, llegó pisando fuerte, tuvo su punto álgido y finalmente se encuentra en decadencia o casi que en extinción, a tal punto que son pocos los que sobreviven hoy en día y sin la posibilidad de ofrecer servicios itinerantes. De las 10 plazas que estaban abiertas en la ciudad hace poco más de un año, solo quedan, al día de hoy, tres plazas de comida food truck (Fudtronomy, Tropique food club, La Plaza), a las que los expertos no le auguran los mejores pronósticos. A continuación, las plazas de comida activas en la ciudad de barranquilla (Tabla $1)$.

Es claro que no nos encontramos ante un simple boom, o un especulativo incremento, del sector de las comidas que va a crecer, estallarse y desinflarse. Este crecimiento tiene fundamentos en cambios socioculturales relacionados con el aumento de las distancias en las ciudades, el crecimiento y agitado ritmo de vida, poco tiempo para cocinar, familias en las que todos trabajan, extensos horarios laborales, cortos tiempos de los horarios de descanso, entre otros. 
Tabla 1. Plazas de comida activas

\begin{tabular}{|c|c|c|c|}
\hline PLAZA DE COMIDA & UBICACIÓN & ESTADO ACTUAL & FOODTRUCKS \\
\hline $\begin{array}{c}\text { THE CONTAINERS } \\
\text { BQ }\end{array}$ & Cra $46 N^{\circ} 85-15$ & Cerrado & $\begin{array}{c}\text { Hamburguesas } \\
\text { Mario Bross Ribs } \\
\text { Frulatto Tropical Drinks } \\
\text { El carrito Sanduchero } \\
\text { Viva mi México } \\
\text { Sal y Parilla } \\
\text { The Burger Box } \\
\text { Sweet Choices } \\
\text { To' en palito } \\
\text { Asia cocina de autor } \\
\text { Oggi Pizza } \\
\text { Bololo } \\
\text { Arepas Kon Ke }\end{array}$ \\
\hline $\begin{array}{c}\text { PLAZA } 96 \text { FOOD } \\
\text { TRUCK }\end{array}$ & Cra 45B con calle 96 & Cerrado & $\begin{array}{c}\text { Bur Dog } \\
\text { Fruta Fresca } \\
\text { Prontto Pizza } \\
\text { El nuevo Shawarma } \\
\text { Jhony Ribs } \\
\text { Salteaditos Gourmet } \\
\text { Memins food truck } \\
\text { Sr wings }\end{array}$ \\
\hline FUDTRONOMY & Cra 59B \# 79-235 & Abierto & $\begin{array}{c}\text { Turco's } \\
\text { El chuzon de Joselo } \\
\text { Puccini fatto in casa } \\
\text { Tokyo station } \\
\text { Qarepa } \\
\text { Formaggio } \\
\text { Bagueton } \\
\text { Bull Burger }\end{array}$ \\
\hline $\begin{array}{c}\text { LA PLAZA DEL } \\
\text { SABOR }\end{array}$ & Calle 80\#47-25 & Abierto & $\begin{array}{c}\text { Burger Bash } \\
\text { Nektar } \\
\text { K-co's tex-mex } \\
\text { Cerveza de la plaza }\end{array}$ \\
\hline $\begin{array}{l}\text { TROPIQUE FOOD } \\
\text { CLUB }\end{array}$ & $\begin{array}{l}\text { Av. Las Dunas Villa } \\
\text { Campestre }\end{array}$ & Abierto & $\begin{array}{l}\text { Take away } \\
\text { Cocteles } \\
\text { Pizza }\end{array}$ \\
\hline
\end{tabular}

Fuente: elaboración propia. 
Cada vez se hace mayor la necesidad de consumir alimentos en la calle, con una tendencia marcada en las horas de almuerzo y la cena, el corto tiempo para consumir estos alimentos, a su vez ha marcado, también, una fuerte tendencia en que estos alimentos sean de preparación más rápida e innovadores en tendencias y modelos.

Según Dinero (2016a):

El sector de alimentos y bebidas es uno de los mercados más dinámicos e importantes para la economía nacional el cual se mantiene en constante evolución y crecimiento. El sector de los restaurantes, catering y bares creció en un 8,2 $\%$, esta cifra, aunque es menor a las registradas para los años 2013 y 2014 cuando aumentó en $8,4 \%$ y $10,1 \%$ respectivamente, es mayor al 4,6 $\%$ correspondiente al año 2015 (p. 1).

Mouthón (2017) afirma que "el sector gastronómico en Colombia mueve unos 38 billones de pesos anuales en ventas y genera más de un millón de empleos en Colombia y uno de sus grandes retos es la formalización" (p. 1).

Garzón (2015) afirma que:

Los Food Trucks a la fecha tienen problemas por invasión al espacio público, lo que les genera inestabilidad. Resulta rentable cuando es posible trabajar todos los días el camión, $60 \%$ de las ventas salen de eventos privados, como conciertos, carreras en el autódromo, ferias y 40 $\%$ de lo que se vende en la calle (p. 1).

Ofrecer los mejores menús, las propuestas más innovadoras, un servicio diferenciado, con la calidad adecuada y a un precio justo, se convierten en grandes retos para un mercado que cada día se vuelve más complejo, exigente y que exige menores tiempos de espera para el cliente, por el agitado ritmo de vida que imponen las ciudades y su continuo crecimiento. Para Dinero (2016b):

Los desafíos van más allá de la manipulación de alimentos e incluyen, además, reclutar el mejor talento humano, comprar o arrendar los mejores sitios del mercado inmobiliario, lograr negociaciones óptimas con los proveedores nacionales o extranjeros y establecer la más sólida cadena de suministros para ser eficientes en este sofisticado mundo (p. 1).

Dinero (2016c) afirma que "ofrecer los mejores menús y un servicio diferenciado son los grandes retos en un mercado complejísimo que pide, además de calidad y atención, precios muy competitivos y tiempos de espera cada vez menores" (p. 1). Según Pastori (2017):

El modelo de negocio de comida de Food Truck que hace referencia a (carros móviles vendiendo alimentos) llega a Colombia hace pocos años dando solución a las necesidades cambiantes del exigente mercado colombiano ofreciendo un valor agregado y diferencial ya que cada propietario da un toque personal a su camión, pues ofreciendo comida rápida en la mayoría de los casos gourmet a precios más asequibles que en restaurantes de manteles tradicionales. Este es un negocio que les ha permitido a los aficionados de la cocina emprender en el mundo culinario (p. 1).

Tratándose de un modelo de negocio de carácter novedoso no existe una legislación actual que lo cobije, siendo este su principal problema. Actualmente existe una asociación en Colombia que se encuentra en Bogotá y que recibe el nombre de "Asociación Colombiana de Food trucks", es una de las pocas asociaciones gremiales que existen sobre el tema, en Bogotá utilizan zonas de parqueo y algunas bahías para evitar problemas con las autoridades correspondientes.

Otro problema al cual se enfrentan los dueños de los food trucks, es la necesidad de la definición específica de su target de mercado. El rápido crecimiento del mercado y la baja investigación en el mismo no han permitido, hasta el momento, tener claramente definido cuál es el público por conquistar y cómo se dirigen hacia el mismo, sus preferencias en el momento del consumo, sus prácticas, y las principales necesidades a satisfacer.

Rueda la Prensa (2018) afirma que:

En Barranquilla hay una propensión progresiva del sector gastronómico, se considera que en los últimos años la región Caribe y particularmente Barranquilla han resaltado por un crecimiento 
superior al doble del promedio nacional, aproximadamente de un $40 \%$. Se calcula que el $75 \%$ de los barranquilleros asevera que sale a comer a restaurantes una o dos veces por semana, lo cual hace que un hogar gaste alrededor del 7,2 $\%$ de su presupuesto en alimentos y bebidas consumidos fuera de la casa. Es significativo tener en cuenta que un $59 \%$ de los barranquilleros desean salir a cenar que a almorzar y especialmente los fines de semana, contrario a Bogotá, donde un $54 \%$ prefieren salir a almorzar, la razón de los anterior podría ser por las condiciones climáticas de cada ciudad (p. 1).

\section{METODOLOGÍA}

Se desarrolló una investigación de tipo mixta, basado en las experiencias propias de comensales que han tenido la oportunidad de visitar los food trucks de la ciudad e implicó la recolección y el análisis de datos cuantitativos y cualitativos. Además, el conocimiento y la percepción de personas expertas locales, conocedoras de temas gastronómicos y en materia de food trucks a nivel nacional, con el fin de comprender este fenómeno en Barranquilla.

El desarrollo metodológico de la investigación se centró en diferentes herramientas de apoyo tales como: grupos focales, cuestionarios y encuestas de forma abierta, entrevistas no estructuradas y observaciones no estructuradas.

\section{Diseño de Entrevista a Comensales}

En el desarrollo de la encuesta se entrevistaron un total de 140 personas distribuidas entre los estratos 4, 5 y 6 en la zona norte de Barranquilla. Esta encuesta fue aplicada voluntariamente a diferentes comensales en las plazas de los food trucks de la ciudad.

Para realizar el diseño y la ejecución de la encuesta a comensales, se definieron unas preguntas para determinar específicamente la población objeto de este estudio:

- Género.

- Edad.

- Estrato socioeconómico.

- Ocupación.

Seguidamente, se estructuraron unas preguntas para conocer las tendencias de consumo y preferencias específicas a la hora de visitar los food trucks en Barranquilla.

- Frecuencia de consumo por fuera de casa.

- Horario de consumo.

- Número de visitas por mes.

- Días de semana que visitan food trucks.

- Tipo de comida que consumen en food trucks.

- Personas con las que visitan los food trucks.

Finalmente, para conocer un poco más sobre la experiencia de consumo de la ciudad se establecieron 10 preguntas con el fin de analizar la satisfacción de los comensales con aspectos relacionados con:

- Productos ofrecidos.

- Atención y servicio.

- Precios.

- Marketing.

- Seguridad.

Las preguntas se clasificaron en una escala de 1 a 5 de la siguiente forma (Tabla 2):

Tabla 2. Escala de preguntas

\begin{tabular}{|l|l|}
\hline 1 & Mucho más bajo de mi nivel deseado \\
2 & Más bajo de mi nivel deseado \\
3 & Igual a mi nivel deseado \\
4 & Supera mi nivel deseado \\
5 & Supera notablemente mi nivel deseable. \\
\hline
\end{tabular}

Fuente: elaboración propia. 


\section{Diseño de Entrevistas semi - estructuradas a Expertos Gastronómicos y dueños de food trucks}

Para la otra parte de la investigación, las entrevistas semi - estructuradas se dividieron de acuerdo con las personas a las cuales se le realizarían cada tipo de entrevistas, se diseñaron de forma independiente teniendo en cuenta los grupos de entrevistados. Se diseñaron entrevistas para los siguientes grupos:

- Propietarios de los food trucks en Barranquilla.

- Expertos gastronómicos como chef y administradores en el área gastronómica.

- Propietarios de reconocidos restaurantes de la ciudad.

- Representantes de asociaciones de food trucks en la ciudad de Bogotá y Medellín.

- Influenciadores gastronómicos.

El criterio para la escogencia de los expertos gastronómicos, se basa principalmente, en personas con amplia experiencia en el sector gastronómico a nivel local, nacional e internacional con reconocida trayectoria y conocedores del tema de food trucks.

\section{RESULTADOS}

\section{Análisis de las experiencias de consumo de comensales que visitan los food truck en Barranquilla}

Sobre las tendencias de consumo en general, el $46 \%$ de los entrevistados afirmó que comía por fuera de casa entre 2 y 3 veces por semana, el $35 \%$ lo hace tan solo 1 vez y el $19 \%$ alcanza a hacerlo, incluso, hasta más de 3 veces a la semana.

Es importante tener en cuenta que un $60 \%$ de los barranquilleros prefiere salir a cenar que a almorzar $\mathrm{y}$, sobre todo, los fines de semana, tal como lo confirmaron las personas entrevistadas, puesto que el $60 \%$ come por fuera a la hora de la cena y solo el 31 $\%$ lo hace a la hora del almuerzo. El desayuno, al igual que el resto de los colombianos, sigue siendo la comida menos preferida para comer fuera de casa, y solo alcanza un $8,6 \%$.
Sobre los food trucks, el $68 \%$ de los entrevistados comen entre 1 y 2 veces al mes en un food truck, y el $25 \%$ de 2 a 4 veces por mes, y únicamente una minoría del $7 \%$ comen más de 4 veces al mes en estos lugares. El 48,6\% prefiere ir a comer con su pareja, mientras que el $39,3 \%$ en familia y un $12 \%$ decide hacerlo solo. Los sábados fueron escogidos por 78 personas y el domingo por 71 , de los 140 entrevistados, como los días preferidos para visitar los food trucks.

Según Nielsen (2017):

Sobre los gustos o preferencias en comida, los
Barranquilleros prefieren en mayor medida la
comida rápida, la italiana, la árabe y la mexicana
en los food trucks y en una menor medida la
comida japonesa. Las personas entrevistadas
alcanzan a comer por fuera hasta de 1 a 2 veces
por semana un $45 \%$, siendo mayor al promedio
nacional, según los resultados de Nielsen que
alcanzan un $38 \%$ (p. 2).

Sobre la experiencia puntual de consumo en los food trucks de Barranquilla, las preguntas se centraron en varios ítems para analizar la satisfacción de los usuarios en estas plazas de comida.

Con respecto a los estándares de los productos ofrecidos en los food trucks, solo el $12 \%$ de los comensales entrevistados consideraron que estaba por debajo del nivel deseado, mientras que cerca del $40 \%$ piensan que cumplen con el nivel esperado y el restante $48 \%$ considera que su experiencia con la calidad de los productos ofrecidos supera el nivel deseado.

Sobre la variedad de los productos, tan solo el $15 \%$ considera que no es suficiente o no cumple con sus expectativas, mientras que el $85 \%$ restante considera que esta igual o por encima del nivel deseado, es decir, encontraban buena la oferta gastronómica en los food trucks.

El $21 \%$ de los entrevistados sienten que no tienen una atención cercana y personalizada en los food trucks, el $38 \%$ considera que cumple con sus expectativas, mientras que el $41 \%$ restante considera que la atención es lo suficientemente buena y cercana y está por encima del nivel esperado o deseado por 
ellos. Así mismo, el $10 \%$ de los comensales de food trucks que se entrevistaron consideran que los horarios no cumplen con el nivel requerido o los horarios deseados por ellos, mientras que el $90 \%$ restante consideran que están igual o por encima del nivel deseado.

En lo referente a los tiempos de entrega del pedido, el $16 \%$ indica que no cumple con sus expectativas, el $50 \%$ de las personas entrevistadas dicen que sí cumplen, y el restante $34 \%$ afirma estar muy satisfecho con los tiempos de entrega. El $28 \%$ de los entrevistados estiman que en los food trucks no hay un sincero interés en resolverle sus problemas, mientras que el restante $72 \%$ se siente satisfecho con respecto a la solución de problemas.

Cerca del $30 \%$ de los entrevistados considera que los precios están muy elevados o no cumplen con el nivel esperado, otro $30 \%$ cree que pagan un precio justo y un $40 \%$, por su parte, se considera muy satisfecho con el precio que le ofrecen en los food trucks.

Sobre las ofertas y promociones, el $29 \%$ de los entrevistados se sienten por debajo del nivel de satisfacción en cuanto a la información que le es suministrada, mientras que el restante $71 \%$ se sienten a gusto con la información sobre promociones y descuentos. Con respecto a las instalaciones solo el $11 \%$ dicen que no cumple con sus expectativas y el restante $89 \%$ las considera igual o por encima del nivel esperado.

La seguridad en los espacios en los que operan los food trucks es un aspecto importante, pues cerca de un $20 \%$ indica no estar satisfecho con el nivel de seguridad ofrecida, mientras que el restante $80 \%$ se siente igual o más a gusto con el nivel deseado de seguridad en los food trucks de la ciudad de Barranquilla.

En términos generales, la experiencia de los asistentes a los food trucks la consideran como positiva y que cumple con sus expectativas, la mayoría aseguraron estar satisfechos con su experiencia, y una minoría manifestaba no estar plenamente agradado.

Para los comensales de los food trucks la propuesta fue buena y cumplía con sus expectativas, pero, a simple vista, no superaban sus expectativas, es decir, cumplían con lo que prometían, sin embargo no generaban una propuesta de valor agregado realmente diferenciadora, lo cual llevara a superar las expectativas de los clientes.

\section{Análisis de la percepción de los propietarios de food trucks}

Todos los propietarios entrevistados concluyeron que la falta de leyes y normas para su regulación, la saturación del mercado, y lo más importante, que personas sin preparación y sin conocimiento previo en el sector gastronómico, se involucraron en este modelo de negocio. Estos hechos ayudaron a que el público Barranquillero desconfiara en la oferta ofrecida al momento de ir a disfrutar en estos lugares. Existió una tendencia facilista de copia de negocio, donde la improvisación en las preparaciones, la comida recalentada, la incomodidad a la hora de comer y los precios altos se convirtieron en factores que determinaron que este modelo de negocio en Barranquilla no fuera exitoso.

Los propietarios de food truck afirman que su inicio fue exitoso, las ventas crecían mes a mes y era una buena oportunidad para darse a conocer, debido a que la ubicación variaba día a día, la gente los buscaba en cualquier lugar. Se le apostó a una buena calidad del producto con un precio excelente, es decir, a lo que es el concepto original de un food truck, vendía comida de calle "barata y abundante".

Como causa de las regulaciones en Barranquilla, con el tema de permisos para transitar en las calles, este modelo de negocio tuvo un cambio, se fueron formando las mal llamadas plaza de comidas de food truck en donde para poder trabajar se necesita parquear el truck pagando administración y servicios. En Barranquilla no existen lugares o zonas que apoyen al emprendedor en este tipo de negocios, como sucede en Medellín y Bogotá que si cuentan con estos espacios.

Lastimosamente están amarrados a muchos gastos administrativos para poder funcionar, sin tener un flujo de gente diario y sin estrategias claras en campañas de mercadeo. Además, nunca se cumplió su expectativa de venta, la idea de este modelo de negocio era comida buena, bonita y barata, donde las cantidades que produjeran generaban una ganancia, por lo menos, aceptable.

Pero a pesar de todas las complicaciones legales, 
logísticas y operativas varios propietarios de food truck, viendo el éxito que esta modalidad tenía, decidieron abrir locales comerciales.

Varios de los trucks han cerrado sus puertas este último año, todo debido a la alta competencia, la tendencia de moda que existe en la ciudad o acosados por los muchos gastos innecesarios que demanda su normal funcionamiento.

Según el Registro Mercantil de la Cámara de Comercio de Barranquilla (CCB) durante el 2016 y los primeros ocho meses del 2017, 181 sociedades inscritas como expendios de comidas preparadas en restaurantes, cafeterías y otras especializadas, no renovaron su matrícula mercantil, es decir, que cerraron su negocio. A raíz de inspecciones de los entes gubernamentales, muchos food trucks han tenido que cerrar por no cumplir con la normatividad, mientras que otros lo han hecho por no poder cumplir las expectativas económicas necesarias para seguir funcionando, puesto que la informalidad ha golpeado fuertemente el sector (Mendoza, 2017).

\section{Análisis de la percepción de los Expertos Gastronómicos}

Todo el análisis se realizó con base en las siguientes preguntas:

- ¿Qué opinión le merece la evolución de la gastronomía en Barranquilla?

- ¿Qué considera que le hace falta al sector gastronómico en Barranquilla?

- ¿Considera que el público Barranquillero es receptivo y abierto a todos los nuevos conceptos que han surgido?

- ¿Qué piensa de la comida como una moda?

- ¿Considera que son los food trucks una moda o acabarán protagonizando un capítulo interesante, pero pasajero en la historia de la comida?

- ¿Qué le espera a Barranquilla en el sector gastronómico?

En cuanto a los food trucks, son una tendencia que se generó por el concepto de la cocina urbana, no es un fenómeno nuestro. Es un negocio para los chefs que no han tenido la oportunidad de tener su propio restaurante. En Barranquilla todo era copiar, copiar y repetir. ¿Cuál era la diferenciación y la innovación en los negocios de los food trucks? ¿Faltaba cercanía con los clientes?

La mayoría de los expertos consideran que no es fenómeno con perdurabilidad, sin embargo, estiman que la experiencia vivida no está perdida, que la industria gastronómica es un negocio, que se aprendieron muchas cosas y las buenas propuestas, que nacieron con el fenómeno de los food trucks en la ciudad, se convertirán muy seguramente en restaurantes.

Empezaron muchos jóvenes a graduarse en las escuelas de gastronomía y quisieron ser chefs de sus propios negocios sin tener experiencia previa, también, muchas personas con algo de capital vieron en los food trucks una buena alternativa con poca inversión, sin embargo, no eran negocios legalmente constituidos, además, en la mayoría de estos no había variedad en la oferta brindada.

Al no poder movilizarse libremente por las calles, el negocio de los food trucks se trasladó a las plazas de comidas, en donde se vivió un fenómeno muy especial en la ciudad, los precios de arriendo eran muy altos y se les salieron de las manos. Además, no es fácil la administración de una cocina de buena calidad en un espacio de $2 \times 2$, eso es algo que necesita gente con amplia experiencia y exigencia.

De modo que, se volvió un negocio inmobiliario y empezaron a cobrar un arriendo mucho más caro que en un centro comercial, con una prima de ingreso alta para entrar a la plaza de comida, sin garantizar un número de clientes por día y sin la libertad de vender lo que se quería, pues sí en la plaza ya había un negocio de pizza, no podía montar otro similar. El modelo estaba destinado al fracaso.

Se convirtió en un negocio para los dueños de los lotes o parqueaderos, y la gastronomía no es solo un producto, es una experiencia que te debe invitar a volver. Elementos como; el clima, el baño, la higiene, el parqueadero, la oferta gastronómica, la facilidad de pago, son factores importantes que afectan el éxito en el sector gastronómico.

Llegó el momento en que no había forma para pagar los costos de los arriendos y para poder subsistir, fue necesario, en algunas ocasiones, disminuir la calidad de los productos, lo cual empezó a generar malas 
experiencias tanto en los food trucks como en las plazas de comida.

El comensal no encontraba, ni buen precio, ni calidad, de modo que el emprendedor no encontraba rentabilidad, no había legislación, ni espacio público, ni movilidad, ni salubridad, era una papa caliente que nadie quería agarrar.

En Barranquilla, podemos decir que los food trucks fueron una moda, que al no cumplir con la esencia de ser móvil e itinerante, fueron un boom que lamentablemente duró muy poco.

Según Artuz (2016):

en el 2014, comenzó la tendencia de los carros de comida rodante que rápidamente al darse cuenta de lo complicado que era rodar por las congestionadas calles de la ciudad, decidieron unirse en un solo lugar era posible darle nacimiento a un modelo de negocio sólido que permite, de paso, ofrecerles a los comensales varias alternativas gastronómicas de alto nivel y en un solo lugar (p. 1).

Y mientras los expertos consideran que los food trucks fueron un fenómeno pasajero en Barranquilla, los augurios en cuanto a gastronomía para la ciudad son muy buenos, cada día se incentiva más el turismo y ya no se muestra solo como una ciudad carnavalera, industrial y comercial.

Para Acosta (2017):

debido al aumento en las horas que pasan las personas fuera del hogar por los horarios laborales y el transporte público, lo que genera se dedique menos tiempo a la actividad de cocinar en el hogar optando por comer en restaurantes (p. 2).

Barranquilla será la ciudad restaurantera de Colombia, después de Bogotá, pues no es tan conservadora, ni regionalista, es multicultural. Así, ¿Cuál es el plato típico de Barranquilla? Solo el arroz de lisa, pero Barranquilla recoge todas las comidas típicas de la costa; el mote de queso de la sabana, la butifarra de soledad, etc.
Silva (2017) afirma que:

estamos en el momento en el que se están consolidando marcas reconocidas por sus buenos precios y buena calidad. El barranquillero es proclive hacia las marcas derivadas de otros idiomas y las recibe de manera positiva por ese antecedente multicultural que se percibe desde hace muchos años en la ciudad (p. 135).

Los barranquilleros cada día están más atrevidos y dispuestos a probar muchas cosas nuevas, seguirán saliendo muchos restaurantes buenos, vienen premios locales, nuevos chefs que ayudarán a consolidar la ciudad en materia gastronómica.

Villafranco (2014) afirma que "los food trucks podrían ser una oportunidad más de negocio para los emprendedores, solo es cuestión que autoridades y contribuyentes, pongan cada uno de su parte para que todo marche sobre ruedas" (p. 1). Cuevas (2014) considera que "es de resaltar que los food trucks ahorran gastos de anuncios publicitarios y se convierten en un servicio de pauta ambulante que comercializa sus productos directamente a sus clientes" (p. 2).

\section{DISCUSIÓN}

Este fenómeno se difundió rápidamente y llegó a la ciudad de Barranquilla donde no pasó desapercibido, llegó pisando fuerte, tuvo su punto álgido $\mathrm{y}$, finalmente, se encuentra en decadencia o casi que en extinción, a tal punto que el único que sobrevive hoy en día es Grosso food truck, pero sin la posibilidad de ser itinerante por las restricciones de ley. Así mismo, de las 10 plazas que estaban abiertas en la ciudad, hace más de 1 año, solo quedan 3; Fudtronomy, Tropique food club y la Plaza, a los cuales los expertos no le aseguran los mejores resultados. Los propietarios de los food trucks coinciden con los expertos gastronómicos en que la falta de regulación legal y la gran saturación del mercado, así como la llegada al negocio de muchas personas sin preparación y sin conocimiento previo en el sector gastronómico, fueron factores determinantes que atentaron contra el éxito de esta actividad gastronómica. Tal y como afirma Pérez (2015) que "montar un food truck no conlleva 
solamente atender a la estética y a una oferta atractiva de comida" (p. 1).

Para Gastronomía internacional (2016) "el peculiar negocio de los food trucks ha crecido de forma espectacular y se ha convertido en una opción real para emprender" (p. 1).

\section{CONCLUSIÓN}

De acuerdo con los comensales entrevistados en la investigación, las personas que visitan los food trucks o éstas plaza de food trucks son; en su mayoría jóvenes entre los 18 y los 35 años de edad, estudiantes y empleados acompañados de amigos, pareja y familia, lo hacen en su mayoría entre 1 a 2 veces al mes. $\mathrm{Su}$ experiencia sobre los productos ofrecidos indica que estos contaban con calidad y variedad, algunas veces superaban sus expectativas, de modo que en términos generales, la oferta gastronómica de los food trucks no era mala.

Como conclusión, consideran los clientes, que esta experiencia fracasó por falta de innovación y que los food truck se convirtieron en un negocio de copiar y repetir, donde no había propuestas diferenciadoras, que surgieron porque estaba de moda hacer algún negocio pero no conocían realmente la esencia del negocio de los food trucks. Además, eran negocios que no estaban legalmente constituidos y manejar una cocina en un espacio $2 \times 2$ no resultaba ser nada fácil, menos sin tener la experiencia del sector gastronómico o por lo menos como manejar una cocina.

Los Barranquilleros empezaron a desconfiar de la oferta de este tipo de lugares y, poco a poco, volvieron a migrar a los acostumbrados establecimientos de comida rápida de confianza, a las plazoletas de comida de los centros comerciales y a los restaurantes de la ciudad. Los dueños de los food trucks coinciden, también con los expertos, en que los altos costos administrativos y las dificultades para la operación de cocinas en espacios reducidos, fueron factores determinantes para su no continuidad.

Algo para tener en cuenta fue que en Barranquilla, los dueños de food trucks, nunca hicieron nada por asociarse o presentar intenciones para regularse, 0 hasta ahora no hay proyectos adelantados frente a los entes gubernamentales para la regulación de estos camiones móviles. Esto a diferencia de ciudades como Medellín, en donde, aunque no existen leyes aún, hay voluntad política y permiten circular tranquilamente a los food trucks y estos pueden trabajar, siempre y cuando se cumplan ciertas condiciones y acuerdos que se les impusieron. Además, le han dado un enfoque social al proyecto de los food truck, buscando mejorar los parques y sitios públicos donde circulan, de manera que contribuyan a formar una mejor sociedad, donde ganen la sociedad, la ciudad, el empresario y el emprendedor del food truck.

\section{Declaración sobre conflicto de interés}

Los autores declaran que las aseveraciones desarrolladas en este artículo, no comprometen a alguna institución en particular y que ninguna entidad ha influido para cambiar valores o generar aseveraciones que los favorezcan; por lo tanto, manifestamos que cada afirmación ha surgido del ejercicio de la investigación y son concernientes de los investigadores.

\section{REFERENCIAS BIBLIOGRÁFICAS}

Acosta, L. (9 de octubre de 2017). El circulo vicioso de la comida. Portafolio. Recuperado de http://blogs.portafolio.co/raddar/2017 /10/09/circulo-vicioso-la-comida/.

Artuz, F. A. (11 de diciembre de 2016). Los 'Food Trucks' de Barranquilla crecen con sus dueños unidos. El Tiempo. Recuperado de http://www.eltiempo.com/colombia/b arranquilla/negocio-de-food-trucksesta-en-auge-en-barranquilla-43128

Barcelona Foodie Guide. (2015). Barcelona Foodie Guide. España, Barcelona: En Tendencias Foodies. Recuperado de: http://bcnfoodieguide.com/new/blog/ origen-de-los-food-trucks-elfenomeno-foodie-por-excelencia/ 
Cuevas, M. (2014). Los “food trucks": un caso llamativo de emprendimiento en Colombia. Questionar: Investigación Específica, 2(1), 157-165. Recuperado de

\section{https://doi.org/10.29097/23461098.1} $\underline{32}$

Díaz, A. (2015). Descubre la historia rodante del foodtruck. Sabrosía. Recuperado de https://www.sabrosia.com/2015/04/d escubre-la-historia-rodante-delfoodtruck/

Dinero. (2016a, agosto). Los retos que generaron innovación en restaurantes bogotanos. Dinero. Recuperado de http://www.dinero.com/pais/articulo/ los-dificiles-retos-de-tener-unrestaurante-y-un-food-truck-enbogota/231375.

Dinero (2016b, diciembre). Comidas rápidas: Una industria con gran apetito. Dinero Recuperado de http://www.dinero.com/edicionimpresa/negocios/articulo/el-negocio-de-lacomida-rapida-en-colombia/240022.

Dinero (2016c, diciembre). La radiografía del mercado de comidas rápidas en Colombia. Dinero. Recuperado de http://www.dinero.com/edicionimpresa/negocios/articulo/como-esel-mercado-de-comida-rapida-encolombia/240021.

Garzón, D. (25 de julio de 2015). Monte un Food Truck con $\$ 60$ millones y ventas diarias desde \$300.000. La República. Recuperado de https://www.larepublica.co/ocio/mon te-un-food-truck-con-60-millones-yventas-diarias-desde-300000-2281486

Gastronomia internacional. (2016). Food trucks, una buena opción para emprender. Colombia, Bogotá: Instituto de formación online. Recuperdo de https://gastronomicainternacional.co $\mathrm{m}$ /articulos-culinarios/todos/foodtrucks-una-buena-opcion-paraemprender

Ramírez, N. (2018). Los foodtrucks de Medellín ruedan hacía la normatividad. Revista La Barra, Edición 97. Recuperado de https://drive.google.com/file/d/1PFd0Y 4tlZu44xqu7UJNqnNL16jiyN76m/view

Mendoza, N. (30 de septiembre de 2017). 181 restaurantes cerraron en 20 meses. El Heraldo. Recuperado de https://www.elheraldo.co/barranquill a/181-restaurantes-cerraron-en-20meses-segun-camcomercio-407545

Mouthón, L. (9 de septiembre de 2017). Sector gastronómico mueve ventas por $\$ 38$ billones anuales. El Heraldo. Recuperado de https://www.elheraldo.co/economia/s ector-gastronomico-mueve-ventas-por38-billones-anuales-421296

Newman, J. (2018). El motor de la gastronomía nómada. Miami, Estados Unidos: LfeStyle. Recuperado de http://www.lifestylemiami.com/\%E2\% 80\%9Cfood-trucks\%E2\%80\%9D-elmotor-de-la-\%E2\%80\%9Cgastronomianomada\%E2\%80\%9D/

Nielsen. (30 de enero de 2017). 38\% de los colombianos come fuera de su hogar una o más veces a la semana. Bogotá, Colombia: Sala de Negocios Nielsen. Recuperado http://www.nielsen.com/co/es/pressroom/2017/38-por-ciento-de-loscolombianos-come-fuera-de-su-hogaruna-o-mas-veces-a-la-semana.html

Pastori. (14 de marzo de 2017).

Emprendimiento sobre ruedas. El Heraldo. 
Recuperado de https://www.elheraldo.co/masnegocios/emprendimiento-sobre-ruedas336856

Pérez, A. (2015, octubre). Food trucks, la historia de los camiones de comidas. DiegoCoquillat. Recuperado de https://www.diegocoquillat.com/foodtrucks-la-historia-de-los-camiones-decomidas/

Rueda la Prensa (2018). Dinámica del sector gastronómico de barranquilla. Recuperado de http://www.ruedalaprensa.com/index. $\mathrm{php/noticias/noticias-}$

3/economia/1787-dinamica-delsector-gastronomico-de-barranquilla

Silva, H. (2017). Ambiente de comercio en Barranquilla: un bosquejo histórico de su desarrollo. Revista Pensamiento y Gestión, 42, 102-139. Doi: http://dx.doi.org/10.14482/pege.42.104 $\underline{65}$

Villafranco, G. (26 de diciembre de 2014). Food Trucks, tendencia culinaria sobre ruedas. México: Forbes. Recuperado de https://www.forbes.com.mx/food-truckstendencia-culinaria-sobre-ruedas/ 